TRANSACTIONS OF THE

AMERICAN MATHEMATICAL SOCIETY

Volume 361, Number 6, June 2009, Pages 3289-3302

S 0002-9947(09)04770-9

Article electronically published on January 26, 2009

\title{
BLOW-UP OF SOLUTIONS OF NONLINEAR PARABOLIC INEQUALITIES
}

\author{
STEVEN D. TALIAFERRO
}

\begin{abstract}
We study nonnegative solutions $u(x, t)$ of the nonlinear parabolic inequalities

$$
a u^{\lambda} \leq u_{t}-\Delta u \leq u^{\lambda}
$$

in various subsets of $\mathbf{R}^{n} \times \mathbf{R}$, where $\lambda>\frac{n+2}{n}$ and $a \in(0,1)$ are constants. We show that changing the value of $a$ in the open interval $(0,1)$ can dramatically affect the blow-up of these solutions.
\end{abstract}

\section{INTRODUCTION}

In this paper, we study nonnegative solutions $u(x, t)$ of the nonlinear parabolic inequalities

$$
a u^{\lambda} \leq u_{t}-\Delta u \leq u^{\lambda}
$$

in various subsets of $\mathbf{R}^{n} \times \mathbf{R}$, where $a \in(0,1)$ is a constant and $n \geq 1$ is an integer.

In order to state our results, we define $|(x, t)|$ for $(x, t) \in \mathbf{R}^{n} \times \mathbf{R}$ by

$$
|(x, t)|=\max \left\{|x|,|t|^{1 / 2}\right\},
$$

where $|x|$ is the usual Euclidean norm of $x$ in $\mathbf{R}^{n}$, and we define

$$
\lambda_{B}= \begin{cases}\frac{n+2}{n}\left(\frac{n}{n-1}\right)^{2} & \text { if } n \geq 2 \\ \infty & \text { if } n=1\end{cases}
$$

Note that $\lambda_{B}>\frac{n+2}{n}$.

Our result on the blow-up at the origin of nonnegative solutions of (1.1) is

Theorem 1. Suppose $\lambda>\frac{n+2}{n}$. Then there exists $a=a(n, \lambda) \in(0,1)$ and $C=$ $C(n, \lambda) \in(1, \infty)$ such that for each continuous function

$$
\varphi:(0,1) \rightarrow(0, \infty) \quad(\text { resp. } \varphi:(-1,0) \rightarrow(0, \infty))
$$

there exists a $C^{\infty}$ positive solution $u(x, t)$ of (1.1) in $\left(\mathbf{R}^{n} \times \mathbf{R}\right)-\{(0,0)\}$ such that

$$
u(0, t) \neq O(\varphi(t)) \quad \text { as } t \rightarrow 0^{+} \quad\left(\text { resp. } t \rightarrow 0^{-}\right)
$$

and $|(x, t)|^{\frac{2}{\lambda-1}} u(x, t)$ is bounded between $1 / C$ and $C$ in the region

$\left(\mathbf{R}^{n} \times \mathbf{R}\right)-\left\{(x, t):|x|^{2} \leq t \leq 1\right\} \quad\left(\right.$ resp. $\left.\left(\mathbf{R}^{n} \times \mathbf{R}\right)-\left\{(x, t):-1 \leq t \leq-|x|^{2}\right\}\right)$.

Theorem 1 is in strong contrast to the following result of Poláčik, Quittner, and Souplet [11, 15].

Received by the editors September 4, 2007.

2000 Mathematics Subject Classification. Primary 35K55, 35B40, 35R45.

(C)2009 American Mathematical Society 
Theorem 2. Suppose $1<\lambda<\lambda_{B}$. Then there exists $a=a(n, \lambda) \in(0,1)$ and $C=C(n, \lambda) \in(1, \infty)$ such that if $u(x, t)$ is a $C^{2,1}$ nonnegative solution of (1.1) in

$$
B_{2}(0) \times(0,2) \quad\left(\text { resp. } B_{2}(0) \times(-2,0)\right),
$$

then $u(x, t) \leq C|t|^{\frac{-1}{\lambda-1}}$ for

$$
(x, t) \in B_{1}(0) \times(0,1) \quad\left(\text { resp. }(x, t) \in B_{1}(0) \times(-1,0)\right) .
$$

Our result on the blow-up at $t= \pm \infty$ of nonnegative solutions of (1.1) is

Theorem 3. Suppose $\lambda>\frac{n+2}{n}$. Then there exists $a=a(n, \lambda) \in(0,1)$ and $C=$ $C(n, \lambda) \in(1, \infty)$ such that for each continuous function

$$
\varphi:(1, \infty) \rightarrow(0, \infty) \quad(\text { resp. } \varphi:(-\infty,-1) \rightarrow(0, \infty))
$$

there exists a $C^{\infty}$ positive solution $u(x, t)$ of (1.1) in $\mathbf{R}^{n} \times \mathbf{R}$ such that

$$
u(0, t) \neq O(\varphi(t)) \quad \text { as } \quad t \rightarrow \infty \quad(\text { resp. } t \rightarrow-\infty)
$$

and

$$
(1+|(x, t)|)^{\frac{2}{\lambda-1}} u(x, t)
$$

is bounded between $1 / C$ and $C$ in the region

$$
\left\{(x, t): t<|x|^{2}\right\} \quad\left(\text { resp. }\left\{(x, t): t>-|x|^{2}\right\}\right) .
$$

Theorem 3 is in strong contrast to the following result of Poláčik, Quittner, and Souplet [11, 15].

Theorem 4. Suppose $1<\lambda<\lambda_{B}$. Then there exists $a=a(n, \lambda) \in(0,1)$ and $C=C(n, \lambda) \in(1, \infty)$ such that if $u(x, t)$ is a $C^{2,1}$ nonnegative solution of (1.1) in

$$
\left\{(x, t): t>|x|^{2}\right\} \quad\left(\text { resp. }\left\{(x, t): t<-|x|^{2}\right\}\right),
$$

then $u(x, t) \leq C|t|^{\frac{-1}{\lambda-1}}$ for

$$
(x, t) \in B_{1}(0) \times(2, \infty) \quad\left(\text { resp. }(x, t) \in B_{1}(0) \times(-\infty,-2)\right) .
$$

When $\frac{n+2}{n}<\lambda<\lambda_{B}$, these four theorems show that changing the value of $a$ in the open interval $(0,1)$ can dramatically affect the blow-up of positive solutions of (1.1).

Theorem 1 is not true when $\lambda \leq \frac{n+2}{n}$. In fact, we prove in [17] that if $u(x, t)$ is a $C^{2,1}$ nonnegative solution of the parabolic inequalities

$$
0 \leq u_{t}-\Delta u \leq u^{\frac{n+2}{n}}+1
$$

in a punctured neighborhood of the origin in $\mathbf{R}^{n} \times[0, \infty)$, then

$$
u(x, t)=O\left(t^{-n / 2}\right) \quad \text { as } \quad(x, t) \rightarrow(0,0), t>0 .
$$

If $\lambda>\frac{n+2}{n}$, then by Theorem 1, there exists $a \in(0,1)$ such that (1.1) has $C^{2,1}$ positive solutions in $B_{1}(0) \times(0,1)$ which are arbitrarily large as $(x, t)$ approaches $(0,0)$ along the positive $t$-axis. Let $I_{1}=I_{1}(n, \lambda)$ be the set of all such $a$.

If $1<\lambda<\lambda_{B}$, then by Theorem 2 , there exists $a \in(0,1)$ such that every $C^{2,1}$ positive solution $u(x, t)$ of (1.1) in $B_{1}(0) \times(0,1)$ satisfies

$$
u(0, t)=O\left(t^{\frac{-1}{\lambda-1}}\right) \quad \text { as } t \rightarrow 0^{+} .
$$

Let $I_{2}=I_{2}(n, \lambda)$ be the set of all such $a$. 
An interesting open question is whether

$$
I_{1}(n, \lambda) \cup I_{2}(n, \lambda)=(0,1) \text { for all } \lambda \in\left(\frac{n+2}{n}, \lambda_{B}\right) \text { and } n \geq 1 .
$$

If not, how do the $C^{2,1}$ positive solutions of (1.1) in $B_{1}(0) \times(0,1)$ behave as $(x, t)$ approaches the origin along the positive $t$-axis when $a \in(0,1)-\left(I_{1} \cup I_{2}\right)$ ? A similar question can be asked about Theorems 3 and 4 . These questions seem to be very difficult.

The blow-up of solutions of the equation

$$
u_{t}-\Delta u=u^{\lambda}
$$

has been extensively studied in [1, 2, 3, 4, 5, 6, 7, 8, 9, 10, 11, 12, 14, 18, and elsewhere. See [13] and [5] for a summary of many of these results. However, other than [15], we know of no previous results for the inequalities (1.1). When $\frac{n+2}{n}<\lambda<\lambda_{B}$, our results show that it is more appropriate to study the inequalities (1.1) rather than the equation (1.3).

An elliptic analog of the results in this paper can be found in [16].

\section{Preliminary Results}

In this section, we introduce some notation and obtain some results that will be used in Sections 3 and 4 to prove Theorems 1 and 3 , respectively.

Lemma 1. Let $f$ be a $C^{\infty}$ nonnegative function in an open subset $\Omega$ of $\mathbf{R}^{n} \times \mathbf{R}$ and define

$$
u(x, t):=\iint_{\Omega} \Phi(x-y, t-s) f(y, s) d y d s \quad \text { for } \quad(x, t) \in \Omega,
$$

where

$$
\Phi(x, t)= \begin{cases}\frac{1}{(4 \pi t)^{n / 2}} e^{-\frac{|x|^{2}}{4 t}} & \text { for } t>0, \\ 0 & \text { for } t \leq 0\end{cases}
$$

is the heat kernel. If $u \in L_{\mathrm{loc}}^{1}(\Omega)$, then $u$ is $C^{\infty}$ in $\Omega$ and $H u=f$ in $\Omega$, where $H u=u_{t}-\Delta u$ is the heat operator.

Proof. Let $\psi \in C_{0}^{\infty}(\Omega)$. Multiplying (2.1) by $H^{*} \psi:=\psi_{t}+\Delta \psi$, integrating the resulting equation over $\Omega$, and using Fubini's theorem and the fact that $H \Phi=\delta$, we see that $H u=f$ in $\mathcal{D}^{\prime}(\Omega)$. Thus by standard parabolic regularity theory, $u \in C^{\infty}(\Omega)$.

If $(x, t),(y, s) \in \mathbf{R}^{n} \times \mathbf{R}$ and $c \in \mathbf{R}$, then it follows from (1.2) that

$$
|(x, t)+(y, s)| \leq|(x, t)|+|(y, s)|
$$

and $\left|\left(c x, c^{2} t\right)\right|=|c||(x, t)|$.

Throughout this section we assume $\lambda>\frac{n+2}{n}$, which implies

$$
n>\frac{2}{\lambda-1} \text { and } 2<\frac{2 \lambda}{\lambda-1}<n+2 .
$$

Define $W:\left(\mathbf{R}^{n} \times \mathbf{R}\right)-\{(0,0)\} \rightarrow \mathbf{R}$ by

$$
W(y, s)=\left(|y|^{4}+s^{2}\right)^{-\frac{1}{2(\lambda-1)}} .
$$


Then $W$ is $C^{\infty}$ on $\left(\mathbf{R}^{n} \times \mathbf{R}\right)-\{(0,0)\}$ and

$$
W(y, s) \sim|(y, s)|^{-\frac{2}{\lambda-1}} \quad \text { for } \quad 0<|(y, s)|<\infty .
$$

(Here and later the notation $X \sim Y$ (resp. $X \lesssim Y$ ) means $\frac{1}{C} Y \leq X \leq C Y$ (resp. $X \leq C Y$ ) for some positive constant $C$ which depends only on $n$ and $\lambda$.)

Define $W_{0}:\left(\mathbf{R}^{n} \times \mathbf{R}\right)-\{(0,0)\} \rightarrow \mathbf{R}$ by

$$
W_{0}=\varphi W
$$

where $\varphi: \mathbf{R}^{n} \times \mathbf{R} \rightarrow[0,1]$ is a $C^{\infty}$ function satisfying $\varphi(y, s)=1$ for $|(y, s)| \leq 1$ and

$$
\varphi(y, s)=0 \text { for }|(y, s)| \geq \sqrt{\frac{3}{2}} .
$$

Define $w, w_{0}:\left(\mathbf{R}^{n} \times \mathbf{R}\right)-\{(0,0)\} \rightarrow \mathbf{R}$ by

$$
w(x, t)=\iint_{\mathbf{R}^{n} \times \mathbf{R}} \Phi(x-y, t-s) W(y, s)^{\lambda} d y d s
$$

and

$$
w_{0}(x, t)=\iint_{\mathbf{R}^{n} \times \mathbf{R}} \Phi(x-y, t-s) W_{0}(y, s)^{\lambda} d y d s .
$$

It follows from (2.3), (2.4), and (2.5) that $w$ and $w_{0}$ are locally bounded in $\left(\mathbf{R}^{n} \times \mathbf{R}\right)-\{(0,0)\}$. Thus by Lemma $1, w$ and $w_{0}$ are $C^{\infty}$ in $\left(\mathbf{R}^{n} \times \mathbf{R}\right)-\{(0,0)\}$, $H w=W^{\lambda}$ and $H w_{0}=W_{0}^{\lambda}$ in $\left(\mathbf{R}^{n} \times \mathbf{R}\right)-\{(0,0)\}$, and

$$
\begin{aligned}
0 \leq w(x, t)-w_{0}(x, t) & \lesssim \int_{|(y, s)| \geq 1} \Phi(x-y, t-s)|(y, s)|^{-\frac{2 \lambda}{\lambda-1}} d y d s \\
& \lesssim 1 \text { for } 0<|(x, t)| \leq 1 .
\end{aligned}
$$

Lemma 2. The functions $w, W, w_{0}$, and $W_{0}$ satisfy

$$
\begin{aligned}
& \frac{w(x, t)}{W(x, t)} \sim 1 \quad \text { for } \quad 0<|(x, t)|<\infty, \\
& \frac{w_{0}(x, t)}{W_{0}(x, t)} \sim 1 \quad \text { for } \quad 0<|(x, t)| \leq 1,
\end{aligned}
$$

and

$$
\frac{w_{0}(x, t)}{|(x, t)|^{-n}} \lesssim 1 \quad \text { and } \quad \frac{W_{0}(x, t)}{|(x, t)|^{-n}} \lesssim 1 \quad \text { for } \quad 1 \leq|(x, t)|<\infty .
$$

Proof. Making in (2.7) the change of variables

$$
\begin{array}{ll}
x=c \xi & t=c^{2} \tau \\
y=c \eta & s=c^{2} \zeta
\end{array} \quad \text { where } c \text { is a positive constant, }
$$

we get

$$
w(x, t)=c^{-\frac{2}{\lambda-1}} w(\xi, \tau) .
$$

Taking $c=|(x, t)|$ in (2.12) we find

$$
|(\xi, \tau)|=\left|\left(\frac{1}{c} x, \frac{1}{c^{2}} t\right)\right|=\frac{1}{c}|(x, t)|=1
$$

and hence (2.9) follows from (2.13) and (2.4). 
It follows from (2.5), (2.4) and (2.8) that for $0<|(x, t)| \leq 1$ we have

$$
\begin{aligned}
\left|\frac{w_{0}(x, t)}{W_{0}(x, t)}-\frac{w(x, t)}{W(x, t)}\right| & =\frac{\left|w_{0}(x, t)-w(x, t)\right|}{W(x, t)} \\
& \lesssim|(x, t)|^{\frac{2}{\lambda-1}} \rightarrow 0 \quad \text { as } \quad|(x, t)| \rightarrow 0 .
\end{aligned}
$$

Thus (2.10) follows from (2.9) and from the continuity and positivity of $w_{0}$ and $W_{0}$ on $0<|(x, t)| \leq 1$.

Taking $c=|(x, t)| \geq 4$ in (2.12) we have that (2.14) holds, $\frac{2}{|(x, t)|} \leq \frac{1}{2}$, and

$$
\begin{aligned}
\frac{w_{0}(x, t)}{|(x, t)|^{-n}} & \lesssim|(x, t)|^{n} \iint_{|(y, s)| \leq 2} \Phi(x-y, t-s)|(y, s)|^{-\frac{2 \lambda}{\lambda-1}} d y d s \\
& =|(x, t)|^{n-\frac{2}{\lambda-1}} \iint_{|(\eta, \zeta)| \leq 2 /|(x, t)|} \Phi(\xi-\eta, \tau-\zeta)|(\eta, \zeta)|^{-\frac{2 \lambda}{\lambda-1}} d \eta d \zeta \\
& \lesssim|(x, t)|^{n-\frac{2}{\lambda-1}} \iint_{|(\eta, \zeta)| \leq 2 /|(x, t)|}|(\eta, \zeta)|^{-\frac{2 \lambda}{\lambda-1}} d \eta d \zeta \sim 1 .
\end{aligned}
$$

Thus the first inequality of (2.11) follows from the continuity of $w_{0}(x, t)$ for $1 \leq$ $|(x, t)| \leq 4$. The second inequality of (2.11) follows from (2.5) and (2.4).

For $0<r \leq \frac{1}{2}$, define $W_{r}: \mathbf{R}^{n} \times \mathbf{R} \rightarrow \mathbf{R}$ by

$$
W_{r}(y, s)=\left(|y|^{4}+s^{2}+r^{4}\right)^{-\frac{1}{2(\lambda-1)}} \varphi(y, s),
$$

where $\varphi$ is the function in (2.5). Then

$$
\begin{aligned}
& W_{r}(y, s) \sim r^{-\frac{2}{\lambda-1}} \quad \text { for } \quad 0 \leq|(y, s)| \leq r, \\
& W_{r}(y, s) \sim W_{0}(y, s) \quad \text { for } \quad r \leq|(y, s)|<\infty .
\end{aligned}
$$

Recall that according to our definition of $X \sim Y$ after equation (2.4), the constants $C$ for the relations (2.15) and (2.16) above and the relations (2.18) and (2.19) below do not depend on $r$.

For $0<r \leq \frac{1}{2}$, define $w_{r}: \mathbf{R}^{n} \times \mathbf{R} \rightarrow \mathbf{R}$ by

$$
w_{r}(x, t)=\iint_{\mathbf{R}^{n} \times \mathbf{R}} \Phi(x-y, t-s) W_{r}(y, s)^{\lambda} d y d s .
$$

It follows from Lemma 1 that $w_{r}$ is $C^{\infty}$ in $\mathbf{R}^{n} \times \mathbf{R}$ and $H w_{r}=W_{r}^{\lambda}$.

Lemma 3. For $0<r \leq \frac{1}{2}$ we have

$$
\frac{w_{r}(x, t)}{W_{r}(x, t)} \sim 1 \quad \text { for } \quad 0 \leq|(x, t)| \leq 1,
$$

and

$$
\frac{w_{r}(x, t)+W_{r}(x, t)}{|(x, t)|^{-n}} \lesssim 1 \quad \text { for } \quad 1 \leq|(x, t)|<\infty .
$$

Proof. It follows from (2.4), (2.5), (2.15), (2.16), and (2.17) that

$$
w_{r}(x, t) \sim I_{r}(x, t)+J_{r}(x, t)+K_{r}(x, t) \quad \text { for } \quad(x, t) \in \mathbf{R}^{n} \times \mathbf{R},
$$


where

$$
\begin{aligned}
I_{r}(x, t) & =r^{-\frac{2 \lambda}{\lambda-1}} \iint_{|(y, s)|<r} \Phi(x-y, t-s) d y d s \\
J_{r}(x, t) & =\iint_{r<|(y, s)|<1} \Phi(x-y, t-s)|(y, s)|^{-\frac{2 \lambda}{\lambda-1}} d y d s \\
K_{r}(x, t) & =\iint_{1<|(y, s)|<2} \Phi(x-y, t-s) \varphi(y, s)|(y, s)|^{-\frac{2 \lambda}{\lambda-1}} d y d s .
\end{aligned}
$$

For $|(x, t)| \leq r \in\left(0, \frac{1}{2}\right]$ we have

$$
\begin{aligned}
\frac{I_{r}(x, t)}{r^{-\frac{2}{\lambda-1}}} & =r^{-2} \iint_{|(y, s)|<r} \Phi(x-y, t-s) d y d s \leq r^{-2} \int_{-r^{2}}^{r^{2}}\left(\int_{y \in \mathbf{R}^{n}} \Phi(x-y, t-s) d y\right) d s \\
& \leq r^{-2} 2 r^{2}=2 .
\end{aligned}
$$

Making in (2.20) the change of variables (2.12) with $c=r \in\left(0, \frac{1}{2}\right]$, we get

$$
\frac{J_{r}(x, t)}{r^{-\frac{2}{\lambda-1}}}=\widehat{J}_{r}\left(\frac{x}{r}, \frac{t}{r^{2}}\right)
$$

where

$$
\widehat{J}_{r}(\xi, \tau)=\iint_{1 \leq|(\eta, \zeta)| \leq \frac{1}{r}} \Phi(\xi-\eta, \tau-\zeta)|(\eta, \zeta)|^{-\frac{2 \lambda}{\lambda-1}} d \eta d \zeta .
$$

For $|(x, t)| \leq r \in\left(0, \frac{1}{2}\right]$ we have $\left|\left(\frac{x}{r}, \frac{t}{r^{2}}\right)\right| \leq 1$. Also

$$
\begin{aligned}
\sup _{|(\xi, \tau)| \leq 1} \widehat{J}_{r}(\xi, \tau) & \leq \sup _{|(\xi, \tau)| \leq 1} \iint_{1 \leq|(\eta, \zeta)| \leq \infty} \Phi(\xi-\eta, \tau-\zeta)|(\eta, \zeta)|^{-\frac{2 \lambda}{\lambda-1}} d \eta d \zeta \\
& =C(n, \lambda)<\infty
\end{aligned}
$$

and

$$
\begin{aligned}
\inf _{|(\xi, \tau)| \leq 1} \widehat{J}_{r}(\xi, \tau) & \geq \inf _{|(\xi, \tau)| \leq 1} \iint_{1<|(\eta, \zeta)|<2} \Phi(\xi-\eta, \tau-\zeta)|(\eta, \zeta)|^{-\frac{2 \lambda}{\lambda-1}} d \eta d \zeta \\
& \geq C(n, \lambda)>0 .
\end{aligned}
$$

For $r \in\left(0, \frac{1}{2}\right]$ and $(x, t) \in \mathbf{R}^{n} \times \mathbf{R}$, we have

$$
\frac{K_{r}(x, t)}{r^{-\frac{2}{\lambda-1}}} \leq \iint_{1<|(y, s)|<2} \Phi(x-y, t-s) d y d s \leq 8 .
$$

Combining (2.21) -(2.25) and using (2.15) we obtain for $r \in\left(0, \frac{1}{2}\right]$ that

$$
\frac{w_{r}(x, t)}{W_{r}(x, t)} \sim \frac{w_{r}(x, t)}{r^{-\frac{2}{\lambda-1}}} \sim 1 \quad \text { for } \quad|(x, t)|<r .
$$


Since for $|(x, t)| \geq r, W_{r}(x, t) \sim W_{0}(x, t)$ and $w_{r}(x, t) \leq w_{0}(x, t)$, it follows from Lemma 2 that to complete the proof of Lemma 3 we only need to show

$$
\frac{w_{r}(x, t)}{W_{r}(x, t)} \gtrsim 1 \quad \text { for } \quad r \leq|(x, t)| \leq 1 .
$$

To do this, we make in (2.20) the change of variables (2.12) with $c=|(x, t)| \in$ $\left[r, \frac{1}{2}\right]$ to get (2.14) and

$$
\begin{aligned}
& \frac{w_{r}(x, t)}{W_{r}(x, t)} \gtrsim \frac{J_{r}(x, t)}{c^{-\frac{2}{\lambda-1}}}=\iiint_{\frac{r}{c} \leq|(\eta, \zeta)| \leq \frac{1}{c}} \Phi(\xi-\eta, \tau-\zeta)|(\eta, \zeta)|^{-\frac{2 \lambda}{\lambda-1}} d \eta d \zeta \\
& \quad \geq \min _{|(\xi, \tau)|=1} \iint_{1 \leq|(\eta, \zeta)| \leq 2} \Phi(\xi-\eta, \tau-\zeta)|(\eta, \zeta)|^{-\frac{2 \lambda}{\lambda-1}} d \eta d \zeta=C(n, \lambda)>0 .
\end{aligned}
$$

Also, for $r \in\left(0, \frac{1}{2}\right]$ and $|(x, t)| \in\left[\frac{1}{2}, 1\right]$ we have

$$
\frac{w_{r}(x, t)}{W_{r}(x, t)} \sim w_{r}(x, t) \gtrsim K_{r}(x, t) \geq C(n, \lambda)>0 .
$$

Relation (2.26) follows from (2.27) and (2.28).

For $0<r \leq \frac{1}{2}$ and $h>0$, define $W_{h, r}: \mathbf{R}^{n} \times \mathbf{R} \rightarrow \mathbf{R}$ by

$$
W_{h, r}(y, s)=h^{-\frac{2}{\lambda-1}} W_{r}\left(\frac{y}{h}, \frac{s}{h^{2}}\right) .
$$

It follows from (2.5), (2.15), (2.16), and (2.6) that

$$
\begin{array}{lll}
W_{h, r}(y, s) \sim(h r)^{-\frac{2}{\lambda-1}} & \text { for } \quad 0 \leq|(y, s)| \leq h r, \\
W_{h, r}(y, s) \sim|(y, s)|^{-\frac{2}{\lambda-1}} & \text { for } \quad h r \leq|(y, s)| \leq h, \\
W_{h, r}(y, s) \sim|(y, s)|^{-\frac{2}{\lambda-1} \varphi} \varphi\left(\frac{y}{h}, \frac{s}{h^{2}}\right) & \text { for } & h \leq|(y, s)| \leq \sqrt{\frac{3}{2}} h, \\
W_{h, r}(y, s)=0 & \text { for } & |(y, s)| \geq \sqrt{\frac{3}{2}} h .
\end{array}
$$

For $0<r \leq \frac{1}{2}$ and $h>0$, define $w_{h, r}: \mathbf{R}^{n} \times \mathbf{R} \rightarrow \mathbf{R}$ by

$$
w_{h, r}(x, t)=\iint_{\mathbf{R}^{n} \times \mathbf{R}} \Phi(x-y, t-s) W_{h, r}(y, s)^{\lambda} d y d s .
$$

Making in (2.34) the change of variables (2.12) with $c=h$ and using (2.17) and (2.29), we get

$$
w_{h, r}(x, t)=h^{-\frac{2}{\lambda-1}} w_{r}\left(\frac{x}{h}, \frac{t}{h^{2}}\right) .
$$

The following lemma follows immediately from Lemma 3 and equations (2.29) and (2.35).

Lemma 4. For $0<r \leq \frac{1}{2}$ and $h>0$ we have

$$
\begin{array}{lll}
\frac{w_{h, r}(x, t)}{W_{h, r}(x, t)} \sim 1 & \text { for } & 0 \leq|(x, t)| \leq h, \\
\frac{w_{h, r}(x, t)+W_{h, r}(x, t)}{h^{n-\frac{2}{\lambda-1}}|(x, t)|^{-n}} \lesssim 1 & \text { for } & h \leq|(x, t)|<\infty .
\end{array}
$$


Define $\widehat{W}, \widehat{w}: \mathbf{R}^{n} \times \mathbf{R} \rightarrow \mathbf{R}$ by

$$
\begin{aligned}
\widehat{W}(y, s) & =\left(|y|^{4}+s^{2}+1\right)^{-\frac{1}{2(\lambda-1)}}, \\
\widehat{w}(x, t) & =\iint_{\mathbf{R}^{n} \times \mathbf{R}} \Phi(x-y, t-s) \widehat{W}(y, s)^{\lambda} d y d s .
\end{aligned}
$$

Then

$$
\widehat{W}(y, s) \sim(1+|(y, s)|)^{-\frac{2}{\lambda-1}} \quad \text { for } \quad(y, s) \in \mathbf{R}^{n} \times \mathbf{R},
$$

which implies

$$
\widehat{W}(y, s) \sim 1 \quad \text { for } \quad 0 \leq|(y, s)| \leq 1
$$

and

$$
\widehat{W}(y, s) \sim|(y, s)|^{-\frac{2}{\lambda-1}} \text { for }|(y, s)| \geq 1
$$

and it follows from (2.3) and Lemma 1 that $\widehat{w}$ is $C^{\infty}$ in $\mathbf{R}^{n} \times \mathbf{R}$ and $H \widehat{w}=\widehat{W}^{\lambda}$.

For $|(x, t)| \geq 1$, we obtain from (2.4) and (2.9) that

$$
|(x, t)|^{\frac{2}{\lambda-1}} \widehat{w}(x, t) \leq|(x, t)|^{\frac{2}{\lambda-1}} w(x, t) \sim \frac{w(x, t)}{W(x, t)} \sim 1
$$

and making the change of variables (2.12) with $c=|(x, t)| \geq 1$ and using (2.14) and (2.38) we get

$$
\begin{aligned}
|(x, t)|^{\frac{2}{\lambda-1}} \widehat{w}(x, t) & \gtrsim|(x, t)|^{\frac{2}{\lambda-1}} \iint_{|(y, s)| \geq 2} \Phi(x-y, t-s) \frac{1}{|(y, s)|^{\frac{2 \lambda}{\lambda-1}}} d y d s \\
& =\iint_{|(\eta, \zeta)| \geq \frac{2}{(x, t) \mid}} \Phi(\xi-\eta, \tau-\zeta) \frac{1}{|(\eta, \zeta)|^{\frac{2 \lambda}{\lambda-1}}} d \eta d \zeta \\
& \geq \min _{|(\xi, \tau)|=1} \iint_{2 \leq|(\eta, \zeta)| \leq 3} \Phi(\xi-\eta, \tau-\zeta) \frac{1}{|(\eta, \zeta)|^{\frac{2 \lambda}{\lambda-1}}} d \eta d \zeta \\
& =C(n, \lambda)>0 .
\end{aligned}
$$

So $\widehat{w}(x, t) \sim|(x, t)|^{-\frac{2}{\lambda-1}}$ for $|(x, t)| \geq 1$, and thus by (2.38),

$$
\left(\frac{\widehat{w}^{\lambda}}{H \widehat{w}}\right)^{1 / \lambda}=\frac{\widehat{w}}{\widehat{W}} \sim 1 \quad \text { in } \quad \mathbf{R}^{n} \times \mathbf{R} .
$$

For $0<r \leq \frac{1}{2}$ and $h>0$, define $V_{h, r}^{+}, V_{h, r}^{-}: \mathbf{R}^{n} \times \mathbf{R} \rightarrow \mathbf{R}$ by

$$
\begin{aligned}
& V_{h, r}^{+}(x, t)=W_{h, r}\left((x, t)-\left(0,2 h^{2}\right)\right), \\
& V_{h, r}^{-}(x, t)=W_{h, r}\left((x, t)+\left(0,2 h^{2}\right)\right) .
\end{aligned}
$$

We abbreviate these last two equations by writing

$$
V_{h, r}^{ \pm}(x, t)=W_{h, r}\left((x, t) \mp\left(0,2 h^{2}\right)\right)
$$

and in what follows we abbreviate other pairs of equations in a similar way.

For $0<r \leq \frac{1}{2}$ and $h>0$, define $v_{h, r}^{ \pm}: \mathbf{R}^{n} \times \mathbf{R} \rightarrow \mathbf{R}$ by

$$
v_{h, r}^{ \pm}(x, t)=\iint_{\mathbf{R}^{n} \times \mathbf{R}} \Phi(x-y, t-s) V_{h, r}^{ \pm}(y, s)^{\lambda} d y d s .
$$


Then

$$
H v_{h, r}^{ \pm}=\left(V_{h, r}^{ \pm}\right)^{\lambda}
$$

and by (2.34)

$$
v_{h, r}^{ \pm}(x, t)=w_{h, r}\left((x, t) \mp\left(0,2 h^{2}\right)\right) .
$$

Thus the following lemma follows directly from Lemma 4.

Lemma 5. For $0<r \leq \frac{1}{2}$ and $h>0$ we have

$$
\begin{array}{lrl}
\frac{v_{h, r}^{ \pm}(x, t)}{V_{h, r}^{ \pm}(x, t)} \sim 1 & \text { for } & \left|(x, t) \mp\left(0,2 h^{2}\right)\right| \leq h, \\
\frac{v_{h, r}^{ \pm}(x, t)+V_{h, r}^{ \pm}(x, t)}{h^{n-\frac{2}{\lambda-1}}\left|(x, t) \mp\left(0,2 h^{2}\right)\right|^{-n}} \lesssim 1 & \text { for } & \left|(x, t) \mp\left(0,2 h^{2}\right)\right| \geq h .
\end{array}
$$

Lemma 5 and equations (2.30), (2.31), (2.4), and (2.9) imply

$$
v_{h, r}^{ \pm} \sim V_{h, r}^{ \pm} \gtrsim h^{-\frac{2}{\lambda-1}} \sim|(x, t)|^{-\frac{2}{\lambda-1}} \sim W \sim w \quad \text { for } \quad\left|(x, t) \mp\left(0,2 h^{2}\right)\right| \leq h .
$$

Since for $\left|(x, t) \mp\left(0,2 h^{2}\right)\right| \geq h$,

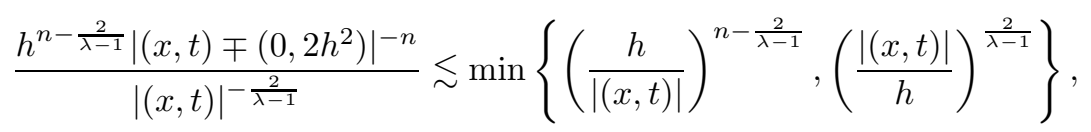

it follows from Lemma 5 that

$$
\frac{v_{h, r}^{ \pm}(x, t)+V_{h, r}^{ \pm}(x, t)}{|(x, t)|^{-\frac{2}{\lambda-1}}} \lesssim \min \left\{\left(\frac{h}{|(x, t)|}\right)^{n-\frac{2}{\lambda-1}},\left(\frac{|(x, t)|}{h}\right)^{\frac{2}{\lambda-1}}\right\}
$$

for $\left|(x, t) \mp\left(0,2 h^{2}\right)\right| \geq h$.

Let $h_{j}=3^{j}$ for $j \in \mathbf{Z}$. Let $\varphi:(-\infty, 0) \cup(0, \infty) \rightarrow(0, \infty)$ be a continuous function. (There is no loss of generality in assuming the functions $\varphi$ in Theorems 1 and 3 are all positive and continuous on the larger set $(-\infty, 0) \cup(0, \infty)$.) Choose $r_{j} \in\left(0, \frac{1}{2}\right]$ such that

$$
\frac{\left(h_{j} r_{j}\right)^{-\frac{2}{\lambda-1}}}{\varphi\left( \pm 2 h_{j}^{2}\right)} \rightarrow \infty \quad \text { as } \quad|j| \rightarrow \infty
$$

Let $V_{j}^{ \pm}=V_{h_{j}, r_{j}}^{ \pm}$and $v_{j}^{ \pm}=v_{h_{j}, r_{j}}^{ \pm}$. Since by (2.33) the support of $V_{j}^{ \pm}$is contained in $R_{\sqrt{\frac{3}{2}} h_{j}}\left(0, \pm 2 h_{j}^{2}\right)$, where

$$
R_{h}(x, y):=\left\{(y, s) \in \mathbf{R}^{n} \times \mathbf{R}:|(y, s)-(x, t)| \leq h\right\},
$$

we see that the functions $V_{j}^{ \pm}, j \in \mathbf{Z}$, have disjoint supports. By Lemma 5 and equation (2.30),

$$
v_{j}^{ \pm}\left(0, \pm 2 h_{j}^{2}\right) \sim V_{j}^{ \pm}\left(0, \pm 2 h_{j}^{2}\right)=W_{h_{j}, r_{j}}(0,0) \sim\left(h_{j}, r_{j}\right)^{-\frac{2}{\lambda-1}}
$$

for $j \in \mathbf{Z}$. 
If $A$ is any subset of $\mathbf{Z}$ and $R_{j}^{ \pm}=R_{h_{j}}\left(0, \pm 2 h_{j}^{2}\right)$ it follows from (2.43) that for $(x, t) \notin \bigcup_{j \in A} R_{j}^{ \pm}$and $(x, t) \neq(0,0)$ we have

$$
\begin{aligned}
\frac{\sum_{j \in A} v_{j}^{ \pm}(x, t)+V_{j}^{ \pm}(x, t)}{|(x, t)|^{-\frac{2}{\lambda-1}}} & \lesssim \sum_{h_{j} \leq|(x, t)|}\left(\frac{h_{j}}{|(x, t)|}\right)^{n-\frac{2}{\lambda-1}}+\sum_{h_{j} \geq|(x, t)|}\left(\frac{|(x, t)|}{h_{j}}\right)^{\frac{2}{\lambda-1}} \\
& \lesssim 1
\end{aligned}
$$

and thus, since the functions $V_{j}^{ \pm}$have disjoint supports,

$$
\frac{\sum_{j \in A} V_{j}^{ \pm}(x, t)^{\lambda}}{|(x, t)|^{-\frac{2 \lambda}{\lambda-1}}}=\left(\frac{\sum_{j \in A} V_{j}^{ \pm}(x, t)}{|(x, t)|^{-\frac{2}{\lambda-1}}}\right)^{\lambda} \lesssim 1
$$

for $(x, t) \notin \bigcup_{j \in A} R_{j}^{ \pm}$and $(x, t) \neq(0,0)$.

\section{Proof of Theorem 1}

In this section, we use the notation and results in Section 2 to prove Theorem 1 .

Proof of Theorem 1. Since the functions $V_{j}^{ \pm}, j \in \mathbf{Z}$, are $C^{\infty}$ and have disjoint support, $\sum_{j \leq-1}\left(V_{j}^{ \pm}\right)^{\lambda}$ converges in $\left(\mathbf{R}^{n} \times \mathbf{R}\right)-\{(0,0)\}$ to a $C^{\infty}$ function. It follows from the monotone convergence theorem and (2.40) that

$$
\begin{aligned}
v^{ \pm}(x, t) & :=\iint_{\mathbf{R}^{n} \times \mathbf{R}} \Phi(x-y, t-s)\left(\sum_{j \leq-1} V_{j}^{ \pm}(y, s)^{\lambda}\right) d y d s \\
& =\sum_{j \leq-1} v_{j}^{ \pm}(x, t) \\
& \lesssim \begin{cases}|(x, t)|^{-\frac{2}{\lambda-1}} & \text { if }(x, t) \notin \bigcup_{j \leq-1} R_{j}^{ \pm}, \\
v_{j_{0}}^{ \pm}(x, t)+|(x, t)|^{-\frac{2}{\lambda-1}} & \text { if }(x, t) \in R_{j_{0}}^{ \pm} \text {for some } j_{0} \leq-1\end{cases}
\end{aligned}
$$

by (2.46). Thus $v^{ \pm}$is bounded on compact subsets of $\left(\mathbf{R}^{n} \times \mathbf{R}\right)-\{(0,0)\}$, and so by Lemma 1, $v^{ \pm}$is $C^{\infty}$ on $\left(\mathbf{R}^{n} \times \mathbf{R}\right)-\{(0,0)\}$ and

$$
H v^{ \pm}=\sum_{j \leq-1}\left(V_{j}^{ \pm}\right)^{\lambda}=\sum_{j \leq-1} H v_{j}^{ \pm}
$$

by (2.41).

Define $u^{ \pm}:\left(\mathbf{R}^{n} \times \mathbf{R}\right)-\{(0,0)\} \rightarrow \mathbf{R}$ by $u^{ \pm}=w+v^{ \pm}$, where $w$ is given by (2.7). Then $u^{ \pm}$is $C^{\infty}$ and

$$
H u^{ \pm}=W^{\lambda}+\sum_{j \leq-1}\left(V_{j}^{ \pm}\right)^{\lambda}
$$

We now show

$$
H u^{ \pm} \sim\left(u^{ \pm}\right)^{\lambda} \quad \text { in } \quad\left(\mathbf{R}^{n} \times \mathbf{R}\right)-\{(0,0)\}
$$

which, after scaling $u^{ \pm}$if necessary, implies $u^{ \pm}$satisfies (1.1) in $\left(\mathbf{R}^{n} \times \mathbf{R}\right)-\{(0,0)\}$. 

(2.46),

If $(x, t) \notin \bigcup_{j \leq-1} R_{j}$ and $(x, t) \neq(0,0)$, then by (3.2), (2.4), (2.9), (2.47), and

$$
\begin{aligned}
H u^{ \pm} & =W^{\lambda}\left[1+\sum_{j \leq-1}\left(\frac{V_{j}^{ \pm}}{W}\right)^{\lambda}\right] \\
& \sim w^{\lambda}\left[1+\sum_{j \leq-1} \frac{\left(V_{j}^{ \pm}\right)^{\lambda}}{|(x, t)|^{-\frac{2 \lambda}{\lambda-1}}}\right] \\
& \sim w^{\lambda} \sim w^{\lambda}\left(1+\left(\frac{v^{ \pm}}{w}\right)^{\lambda}\right)=\left(u^{ \pm}\right)^{\lambda} .
\end{aligned}
$$

If $(x, t) \in R_{j_{0}}$ for some $j_{0} \leq-1$, then by (3.2), (2.42), (2.46), and Lemma 5 ,

$$
\begin{aligned}
H u^{ \pm} & =\left(V_{j_{0}}^{ \pm}\right)^{\lambda}+W^{\lambda} \\
& =\left(V_{j_{0}}^{ \pm}\right)^{\lambda}\left[1+\left(\frac{W}{V_{j_{0}}^{ \pm}}\right)^{\lambda}\right] \\
& \sim\left(V_{j_{0}}^{ \pm}\right)^{\lambda} \sim\left(v_{j_{0}}^{ \pm}\right)^{\lambda} \\
& \sim\left(v_{j_{0}}^{ \pm}\right)^{\lambda}\left[1+\sum_{\substack{j \leq-1 \\
j \neq j_{0}}} \frac{v_{j}^{ \pm}}{v_{j_{0}}^{ \pm}}+\frac{w}{v_{j_{0}}^{ \pm}}\right]^{\lambda} \\
& =\left(u^{ \pm}\right)^{\lambda},
\end{aligned}
$$

which proves (3.3).

It follows from (2.44) and (2.45) that $u^{ \pm}(0, t) \neq O(\varphi(t))$ as $t \rightarrow 0^{ \pm}$.

By (2.4), (2.9), and (3.1) we see that $|(x, t)|^{\frac{2}{\lambda-1}} u^{ \pm}(x, t) \sim 1$ in the regions stated in Theorem 1 .

Taking $u=u^{+}$(resp. $u=u^{-}$), we obtain Theorem 1,

\section{Proof of Theorem 3}

In this section, we use the notation and results in Section 2 to prove Theorem 3 , Proof of Theorem [3. Since the functions $V_{j}^{ \pm}, j \in \mathbf{Z}$, are $C^{\infty}$ and have disjoint support, $\sum_{j \geq 1}\left(V_{j}^{ \pm}\right)^{\lambda}$ converges on $\mathbf{R}^{n} \times \mathbf{R}$ to a $C^{\infty}$ function.

Let $B$ be a subset of $\mathbf{N}$. If $|(x, t)|<1$, then $(x, t) \notin \bigcup_{j \in B} R_{j}^{ \pm}$and it therefore follows from (2.43) that

$$
\sum_{j \in B} v_{j}^{ \pm}(x, t)+V_{j}^{ \pm}(x, t) \lesssim \sum_{j \geq 1} h_{j}^{-\frac{2}{\lambda-1}} \sim 1
$$

Thus, by (2.46), we have for $(x, t) \notin \bigcup_{j \in B} R_{j}^{ \pm}$that

$$
\sum_{j \in B} v_{j}^{ \pm}(x, t)+V_{j}^{ \pm}(x, t) \lesssim(1+|(x, t)|)^{-\frac{2}{\lambda-1}}
$$


Hence, since the functions $V_{j}^{ \pm}$have disjoint support,

$$
\sum_{j \in B} V_{j}^{ \pm}(x, t)^{\lambda} \lesssim(1+|(x, t)|)^{-\frac{2 \lambda}{\lambda-1}}
$$

for $(x, t) \notin \bigcup_{j \in B} R_{j}^{ \pm}$.

It follows from the monotone convergence theorem and (2.40) that

$$
\begin{aligned}
v^{ \pm}(x, t) & :=\iint_{\mathbf{R}^{n} \times \mathbf{R}} \Phi(x-y, t-s) \sum_{j \geq 1} V_{j}^{ \pm}(y, s)^{\lambda} d y d s \\
& =\sum_{j \geq 1} v_{j}^{ \pm}(x, t) \\
& \lesssim \begin{cases}(1+|(x, t)|)^{-\frac{2}{\lambda-1}} & \text { if }(x, t) \notin \bigcup_{j \geq 1} R_{j}^{ \pm}, \\
v_{j_{0}}(x, t)+(1+|(x, t)|)^{-\frac{2}{\lambda-1}} & \text { if }(x, t) \in R_{j_{0}}^{ \pm} \text {for some } j_{0} \geq 1\end{cases}
\end{aligned}
$$

by (4.1). Thus $v^{ \pm}$is bounded on compact subsets of $\mathbf{R}^{n} \times \mathbf{R}$ and so by Lemma 1, $v^{ \pm}$is $C^{\infty}$ in $\mathbf{R}^{n} \times \mathbf{R}$ and

$$
H v^{ \pm}=\sum_{j \geq 1}\left(V_{j}^{ \pm}\right)^{\lambda}=\sum_{j \geq 1} H v_{j}^{ \pm}
$$

by (2.41).

Define $u^{ \pm}: \mathbf{R}^{n} \times \mathbf{R} \rightarrow \mathbf{R}$ by $u^{ \pm}=\widehat{w}+v^{ \pm}$, where $\widehat{w}$ is given by (2.36). Then $u^{ \pm}$ is $C^{\infty}$ and

$$
H u^{ \pm}=\widehat{W}^{\lambda}+\sum_{j \geq 1}\left(V_{j}^{ \pm}\right)^{\lambda}
$$

We now show

$$
H u^{ \pm} \sim\left(u^{ \pm}\right)^{\lambda} \quad \text { in } \quad \mathbf{R}^{n} \times \mathbf{R},
$$

which, after scaling $u^{ \pm}$if necessary, implies $u^{ \pm}$satisfies (1.1) in $\mathbf{R}^{n} \times \mathbf{R}$.

If $(x, t) \notin R_{j}^{ \pm}$, then by (4.4), (4.2), (2.37), (2.39), and (4.1), we have

$$
\begin{aligned}
H u^{ \pm} & =\widehat{W}^{\lambda}\left[1+\sum_{j \geq 1}\left(\frac{V_{j}^{ \pm}}{\widehat{W}^{\prime}}\right)^{\lambda}\right] \\
& \sim \widehat{w}^{\lambda} \sim \widehat{w}^{\lambda}\left[1+\left(\frac{v^{ \pm}}{\widehat{w}}\right)\right]^{\lambda}=\left(u^{ \pm}\right)^{\lambda} .
\end{aligned}
$$

If $(x, t) \in R_{j_{0}}^{ \pm}$for some $j_{0} \geq 1$, then $|(x, t)| \geq 1$ and so (2.42), (2.37), and (2.39) imply

$$
v_{j_{0}}^{ \pm} \sim V_{j_{0}}^{ \pm} \geq h_{j_{0}}^{-\frac{2}{\lambda-1}} \sim|(x, t)|^{-\frac{2}{\lambda-1}} \sim(1+|(x, t)|)^{-\frac{2}{\lambda-1}} \sim \widehat{W} \sim \widehat{w}
$$


Hence, if $(x, t) \in R_{j_{0}}^{ \pm}$for some $j_{0} \geq 1$, then by (4.4), (4.1) and Lemma 5, we have

$$
\begin{aligned}
H u^{ \pm} & =\left(V_{j_{0}}^{ \pm}\right)^{\lambda}+\widehat{W}^{\lambda}=\left(V_{j_{0}}^{ \pm}\right)^{\lambda}\left[1+\left(\frac{\widehat{W}}{V_{j_{0}}^{ \pm}}\right)^{\lambda}\right] \\
& \sim\left(V_{j_{0}}^{ \pm}\right)^{\lambda} \sim\left(v_{j_{0}}^{ \pm}\right)^{\lambda} \sim\left(v_{j_{0}}^{ \pm}\right)^{\lambda}\left[1+\sum_{\substack{j \geq 1 \\
j \neq j_{0}}} \frac{v_{j}^{ \pm}}{v_{j_{0}}^{ \pm}}+\frac{\widehat{w}}{v_{j_{0}}^{ \pm}}\right]^{\lambda} \\
& =\left(u^{ \pm}\right)^{\lambda},
\end{aligned}
$$

which proves (4.5).

It follows from (2.44) and (2.45) that $u^{ \pm}(0, t) \neq O(\varphi(t))$ as $t \rightarrow \pm \infty$. By (2.37), (2.39), and (4.3) we see that $(1+|(x, t)|)^{\frac{2}{\lambda-1}} u^{ \pm}(x, t) \sim 1$ in the regions stated in Theorem 3 .

Taking $u=u^{+}$(resp. $u=u^{-}$), we obtain Theorem 3 ,

\section{Proofs of Theorems 2 and 4}

Souplet [15] showed that the proof of Theorem 3.1 in [11] can be very slightly modified to prove the following theorem.

Theorem 5. Suppose $1<\lambda<\lambda_{B}$ and $D$ is a proper open subset of $\mathbf{R}^{n} \times \mathbf{R}$. Then there exists $a=a(n, \lambda) \in(0,1)$ and $C=C(n, \lambda) \in(1, \infty)$ such that if $u(x, t)$ is a $C^{2,1}$ nonnegative solution of (1.1) in $D$, then

$$
u(x, t) \leq C\left(\inf _{(y, s) \in \partial D}|(y, s)-(x, t)|\right)^{\frac{-2}{\lambda-1}} \quad \text { for all }(x, t) \in D .
$$

Theorems 2 and 4 are immediate consequences of Theorem 5 .

The proofs of Theorem [5 and [11, Theorem 3.1] rely heavily on the following Liouville-type result of Bidaut-Véron [3].

Theorem 6. Suppose $1<\lambda<\lambda_{B}$. Then the only $C^{2,1}$ nonnegative solution $u(x, t)$ of

is $u \equiv 0$.

$$
u_{t}-\Delta u=u^{\lambda} \quad \text { in } \quad \mathbf{R}^{n} \times \mathbf{R}
$$

\section{REFERENCES}

[1] D. Andreucci and E. DiBenedetto, On the Cauchy problem and initial traces for a class of evolution equations with strongly nonlinear sources, Ann. Scuola Norm. Sup. Pisa Cl. Sci. (4) 18, 363-441 (1991). MR.1145316 (92m:35146)

[2] D. Andreucci, M. A. Herrero, and J. J. L. Velázquez, Liouville theorems and blow up behaviour in semilinear reaction diffusion systems, Ann. Inst. H. Poincaré Anal. Non Linéaire 14, 1-53 (1997). MR:1437188 (98e:35088)

[3] M.-F. Bidaut-Véron, Initial blow-up for the solutions of a semilinear parabolic equation with source term. Équations aux dérivées partielles et applications, 189-198, Gauthier-Villars, Ed. Sci. Méd. Elsevier, Paris, 1998. MR1648222 (99h:35082)

[4] Y. Giga and R. V. Kohn, Characterizing blowup using similarity variables, Indiana Univ. Math. J. 36 (1987), 1-40. MR876989 (88c:35021)

[5] Y. Giga, S. Matsui, and S. Sasayama, Blow up rate for semilinear heat equations with subcritical nonlinearity, Indiana Univ. Math. J. 53 (2004), 483-514. MR2060042(2005g:35153) 
[6] M. A. Herrero and J. J. L. Velázquez, Explosion de solutions d'équations paraboliques semilinéaires supercritiques, C. R. Acad. Sci. Paris Sér. I Math. 319 (1994), 141-145. MR.1288393 (95i:35037)

[7] O. Kavian, Remarks on the large time behaviour of a nonlinear diffusion equation, Ann. Inst. H. Poincaré Anal. Non Linéaire 4 (1987), 423-452. MR921547 (89b:35013)

[8] H. Matano and F. Merle, On nonexistence of type II blowup for a supercritical nonlinear heat equation, Comm. Pure Appl. Math. 57 (2004), 1494-1541. MR.2077706 (2005e:35115)

[9] F. Merle and H. Zaag, Optimal estimates for blowup rate and behavior for nonlinear heat equations, Comm. Pure Appl. Math. 51 (1998), 139-196. MR1488298 (98k:35107)

[10] N. Mizoguchi, Type-II blowup for a semilinear heat equation, Adv. Differential Equations 9 (2004), 1279-1316. MR2099557 (2005k:35204)

[11] P. Poláčik, P. Quittner, and P. Souplet, Singularity and decay estimates in superlinear problems via Liouville-type theorems. Part II: Parabolic equations, Indiana Univ. Math. J. 56 (2007), 879-908. MR2317549

[12] P. Poláčik and E. Yanagida, On bounded and unbounded global solutions of a supercritical semilinear heat equation, Math. Ann. 327 (2003), 745-771. MR2023315 (2005b:35133)

[13] P. Quittner and P. Souplet, Superlinear parabolic problems. Blow-up, global existence and steady states, Birkhäuser, Basel, 2007. MR2346798 (2008f:35001)

[14] P. Quittner, P. Souplet, and M. Winkler, Initial blow-up rates and universal bounds for nonlinear heat equations, J. Differential Equations 196, 316-339 (2004). MR2028111|(2005a:35143)

[15] P. Souplet, personal communication.

[16] S. Taliaferro, Local behavior and global existence of positive solutions of $a u^{\lambda} \leq-\Delta u \leq u^{\lambda}$, Ann. Inst. H. Poincaré Anal. Non Linéaire 19 (2002), 889-901. MR1939089 (2003j:35110)

[17] S. Taliaferro, Isolated singularities of nonlinear parabolic inequalities, Math. Ann. 338 (2007), 555-586. MR2317931

[18] L. Véron, Singularities of solutions of second order quasilinear equations. Pitman Research Notes in Mathematics Series, 353. Longman, Harlow, 1996. MR/1424468 (98b:35053)

Department of Mathematics, Texas A\&M University, College Station, Texas 778433368

E-mail address: stalia@math.tamu.edu 